\title{
Effects of 7-nitroindazole, a neuronal nitric oxide synthase (nNOS) inhibitor, on locomotor activity and contextual fear conditioning in rats ${ }^{1}$
}

\author{
Stephen Maren * \\ Department of Psychology and Neuroscience Program, University of Michigan, 525 E. University Avenue, Ann Arbor, MI 48109-1109, USA
}

Accepted 11 June 1998

\begin{abstract}
The effect of 7-nitroindazole (7-NI), a selective neuronal nitric oxide synthase (nNOS) inhibitor, on contextual fear conditioning in rats was examined. Systemic administration of 7-NI did not affect the acquisition of contextual fear (measured as freezing), but it did reduce locomotor activity and cause a corresponding increase in the expression of contextual freezing. It is concluded that nNOS activity is not required for either the acquisition or expression of contextual fear conditioning. () 1998 Elsevier Science B.V. All rights reserved.
\end{abstract}

Keywords: 7-Nitroindazole; Pavlovian conditioning; Learning; Memory; Rat

Nitric oxide (NO) has been suggested to play an important role in both synaptic plasticity and learning and memory, e.g. [16]. For instance, inhibitors of nitric oxide synthase (NOS), the enzyme responsible for generating NO, impair the acquisition of Pavlovian eyeblink conditioning in rabbits [8], passive avoidance conditioning in rats [4], and spatial learning in rats [6,8]; c.f. [1]. Under certain conditions, NOS inhibitors also prevent the induction of hippocampal long-term potentiation (LTP) [7,14,31,32]; c.f. [2,3] and cerebellar long-term depression (LTD) [9], two forms of synaptic plasticity thought to play a role in learning and memory [5,21].

Recent pharmacological studies have implicated the neuronal NOS (nNOS) isoform in synaptic plasticity and learning in rats. Specifically, 7-nitroindazole (7-NI), a selective inhibitor of nNOS that does not produce many of the adverse side effects associated with other NOS inhibitors [27], has been reported to prevent both hippocampal LTP induction in vivo [10] and spatial learning in the Morris water maze [17]. This supports the view that nNOS is required for both hippocampal LTP and forms of learning and memory that require the hippocampus. Of course, systemic administration of 7-NI also produce inhibition of nNOS in structures other than the hippocampus, such as the cerebellum [20], and would be expected to impair

\footnotetext{
* Tel.: + 1-734-936-6532; Fax: + 1-734-763-7480; E-mail: maren@umich.edu; url: http:/ /www-personal.umich.edu / maren.

${ }^{1}$ Published on the World Wide Web on 24 July 1998.
}

forms of learning that depends on nNOS activity in these structures.

One form of learning that may require the hippocampus is contextual fear conditioning in rats. In the contextual fear conditioning task, rats are placed in a novel chamber and given a few unsignaled footshocks. When returned to the chamber, the rats exhibit a conditional fear response characterized by freezing (somatomotor immobility except for that necessitated by breathing). Contextual fear conditioning that is established with unsignaled footshocks is impaired by either pre-training electrolytic [19,25], c.f. [28] or post-training neurotoxic lesions of the dorsal hippocampus [26]. Moreover, contextual fear conditioning may require hippocampal LTP [13,21]. Contextual fear conditioning is blocked by $N$-methyl-D-aspartate (NMDA) receptor antagonists [18], is augmented by manipulations that enhance LTP induction [22,23], and covaries with sex differences in hippocampal LTP [24]. To examine the role nNOS plays in contextual fear conditioning, I examined whether systemic 7-NI administration affects the acquisition and expression of contextual freezing in rats. Systemic 7-NI $(30 \mathrm{mg} / \mathrm{kg})$ was administered either before conditioning, before testing, or before both conditioning and testing to control for possible state-dependent effects of systemic 7-NI on the acquisition and expression of conditional fear. This single dose of 7-NI was chosen because it inhibits both hippocampal LTP induction in vivo [10] and spatial learning [17], and produces an $85 \%$ inhibition of nNOS activity [20]; higher doses of 7-NI were not used because they decrease pain sensitivity [27]. 
The subjects were 24 adult male Long-Evans rats (300-500 g) born and reared in the Department of Psychology vivarium at the University of California, Los Angeles. After weaning, the rats were group housed in same sex cohorts. At the beginning of the experiment, the rats were individually housed in standard stainless-steel hanging cages on a 14:10-h light-dark cycle (lights on at 7:00 am) and had free access to food and tap water. After individual housing, the rats were handled daily (10-20-s per rat) for five days to acclimate them to the experimenter.

Four identical observation chambers $(28 \times 21 \times 22 \mathrm{~cm}$; Lafayette Instrument, North Lafayette, IN) were used for both conditioning and contextual fear testing. The chambers were constructed from aluminum (sidewalls) and Plexiglas (rear wall, ceiling, and hinged front door). The chambers were situated in chests located in a brightly lit and isolated room. A videocamera placed in front of the observation chambers allowed each subjects' behavior to be observed and recorded by an experimenter in an adjacent room. The floor of each chamber consisted of 18 stainless steel rods (4-mm diameter) spaced $1.5 \mathrm{~cm}$ apart (center-to-center). The rods were wired to a shock generator and scrambler (Lafayette Instrument, North Lafayette, IN) for the delivery of footshock USs. The chambers were cleaned with a 5\% ammonium hydroxide solution, and stainless steel pans containing a thin film of the same solution were placed underneath the grid floors before rats were placed inside. Background noise (70 dB, A-scale) was supplied by ventilation fans in each chest and adjacent shock scramblers.

7-nitroindazole (7-NI, $30 \mathrm{mg} / \mathrm{ml}$; Sigma Chemical, St. Louis, MO) was prepared in $100 \%$ dimethyl sulfoxide (DMSO; Sigma Chemical, St. Louis, MO). On the conditioning day, the rats were randomly assigned to each cell of the $2 \times 2$ design, which had factors of training treatment (2 levels; 7-NI and DMSO) and testing treatment (2 levels, 7 -NI and DMSO). This yielded four groups $(N=6$ per group): DMSO/DMSO, 7-NI/DMSO, DMSO/7-NI, 7NI/7-NI. Thirty minutes prior to conditioning, rats received an intraperitoneal injection of either 7-NI (30 $\mathrm{mg} / \mathrm{kg}$ ) or DMSO. The drug dose and administration parameters were used because they block hippocampal LTP in vivo [10] and spatial learning [17], and produce an $85 \%$ inhibition of nNOS activity [20]. For fear conditioning, the rats were placed in the conditioning chambers in squads of four rats; the chamber position was counterbalanced for each squad and group. The rats received three unsignaled footshocks (2-s, 0.5-mA; 64-s intershock interval) 3 minutes after being placed in the chambers. Sixtyfour seconds following the final shock, the rats were returned to their home cages. Locomotor activity was measured during the 3-min pre-shock period by counting chamber crossovers. A crossover was defined as movement of the rat from one side of the chamber to the other (all four paws were required to cross the chamber's mid- line); only forward locomotion was scored as a crossover (i.e. a crossover was not scored if the rat backed into one side of the chamber from the other).

Twenty-four hours after training fear conditioning to the context was assessed by returning the rats to the conditioning chambers and scoring freezing during an 512-s extinction test. Freezing is an associative fear response evoked by stimuli associated with aversive consequences [12]. Briefly, an observer who was blind to the experimental conditions scored each rat for freezing every $8 \mathrm{~s}$ for a total of 64 observations per rat. Thirty minutes prior to the extinction test half of the rats were injected with either 7-NI or DMSO.

The freezing data were transformed to a percentage of total observations, a probability estimate that is amenable to analysis with parametric statistics. These probability estimates of freezing were analyzed using analysis of variance (ANOVA). Planned comparisons in the form of Fisher LSD tests were performed following a significant omnibus $F$-ratio. All data are represented as means \pm the standard errors of the means (SEMs).

Prior to the delivery of footshock on the conditioning day, rats treated with 7-NI exhibited a marked reduction in locomotor activity. As shown in Fig. 1A, this was manifest in a significant decrease in the number of chamber crossovers during the 3-min pre-shock period. A one-way ANOVA with a factor of training treatment (2 levels, DMSO or 7-NI) revealed a significant main effect of treatment $(F(1,22)=10.7, p<.01)$. Similar decreases in locomotor activity have been reported with 7-NI administration in mice [11] and following treatment with other nitric oxide synthase inhibitors in rats [30]. Thus, the reduction in locomotor activity produced by 7-NI treatment suggests that the administered dose was effective in producing nNOS inhibition.

Freezing observations on the extinction test were averaged over the 512-s test and are shown in Fig. 1B. Examination of Fig. 1B reveals that 7-NI treatment before conditioning did not affect conditional freezing during the subsequent extinction test. That is, rats receiving 7-NI before conditioning and DMSO before extinction testing (7-NI/DMSO) showed levels of freezing that were not significantly different from DMSO/DMSO controls. However, 7-NI treatment before extinction testing significantly increased freezing during the test, regardless of whether training was conducted following 7-NI or DMSO administration. That is, rats in the DMSO/7-NI and 7-NI/7-NI groups showed equivalent and high levels of freezing compared to rats in the DMSO/DMSO and 7-NI/DMSO groups. These observations were confirmed in a two-way ANOVA with factors of training treatment (7-NI, DMSO) and testing treatment (7-NI, DMSO). The ANOVA indicated a significant main effect of testing treatment $(F(1$, $20)=5.8, p<.03)$, and a non-significant main effect of training treatment, $(F(1,20)<1, p=.89)$. The training $\times$ testing interaction was not significant $(F(1,20)<1, p=$ 
(A) Pre-shock motor activity

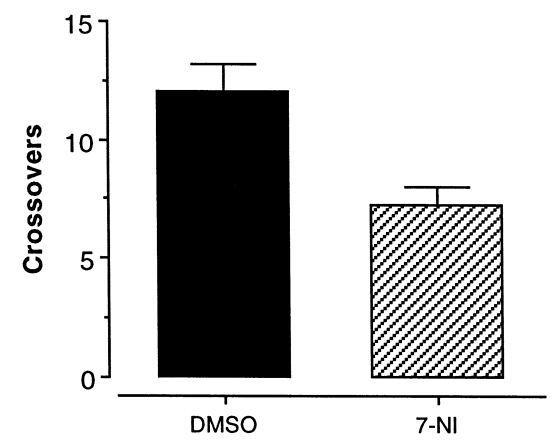

(B) Context extinction test

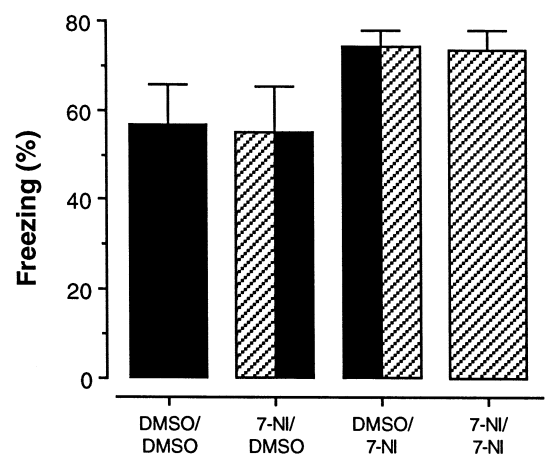

Fig. 1. (A) Mean chamber crossovers $( \pm$ SEM) during the 3-min pre-shock period on the conditioning day for rats receiving DMSO and 7-NI. (B) Mean percentage of freezing ( \pm SEM) collapsed across the 512-s context extinction test for rats receiving DMSO before training and testing (DMSO/DMSO), rats receiving 7-NI before training and DMSO before testing (7-NI/DMSO), rats receiving DMSO before training and 7-NI before testing (DMSO/7-NI), and rats receiving 7-NI before both training and testing (7-NI/7-NI).

.95). These results suggest that 7-NI augments the performance of conditional freezing, but does not affect its acquisition.

The present results indicate that 7-NI, a potent and selective inhibitor of nNOS, does not affect the acquisition of contextual fear conditioning, a form of learning that presumably depends on both the hippocampus and hippocampal LTP. Surprisingly, 7-NI enhanced performance of the freezing response during the extinction test, although this effect can be explained by the nonassociative decrease in locomotor activity produced by 7-NI. Accordingly, these data indicate that nNOS activity is not required for either the acquisition or expression of contextual fear conditioning.

Reports that posit a role for hippocampal LTP in the acquisition of contextual fear conditioning, e.g. [13,21,23$25]$, predict that inhibitors of hippocampal LTP, such as 7-NI, should impair the acquisition of contextual freezing. Clearly, this prediction was not born out in the present study. A number of factors might account for the inability of 7-NI to inhibit contextual fear conditioning. First, it is possible that 7-NI inhibits LTP induction at some hip- pocampal synapses, but leaves LTP at other synapses intact. It has been reported that 7-NI inhibits LTP induction in hippocampal area CA1, but it is not known if 7-NI inhibits LTP induction in the dentate gyrus, for example. This may be an important consideration, because Bannerman and colleagues [2] have found that LTP induction in the dentate gyrus is not blocked by L-arginine methyl ester (L-NAME), another NOS inhibitor. It is also the case that context conditioning can be acquired in the absence of an intact hippocampus [26,28].

Another possibility that should be considered is that multiple forms of NOS may be involved in the induction of hippocampal LTP. For example, it has recently been reported that deletion of both neuronal and endothelial forms of NOS is required to prevent hippocampal LTP induction in vitro [29]. Thus, the selectivity of 7-NI for nNOS may allow LTP induction through the alternate endothelial NOS (eNOS) pathway. However, 7-NI has been found to block LTP in hippocampal area CA1 in vivo, suggesting that the eNOS pathway is not sufficient for LTP induction in this brain region. Further work is clearly required to determine the role various forms of NOS play in LTP induction.

Other factors to consider are that the vehicle for 7-NI delivery and the strain of rats used in the present study were different from that used in previous experiments $[10,17]$. Nevertheless, the present data suggest that two forms of learning that are believed to require hippocampal LTP, that is spatial learning and contextual conditioning, are differentially sensitive to nNOS inhibition. This provides further support for the view that these forms of learning may, in fact, be mediated by different neurobiological mechanisms, e.g. [15].

\section{Acknowledgements}

I would like to thank Gal Aharonov for assistance in running the experiment and Dr. Terry Robinson for helpful comments on a draft of the manuscript. The research reported here was conducted while the author was at the University of California, Los Angeles and was supported by a National Research Service Award (MH11061) from the National Institute of Mental Health and a National Institute of Mental Health Grant (MH39786) to Dr. Michael S. Fanselow.

\section{References}

[1] D.M. Bannerman, P.F. Chapman, P.A.T. Kelly, S.P. Butcher, R.G.M. Morris, Inhibition of nitric oxide synthase does not impair spatial learning, J. Neurosci. 14 (1994) 7404-7414.

[2] D.M. Bannerman, P.F. Chapman, P.A.T. Kelly, S.P. Butcher, R.G.M. Morris, Inhibition of nitric oxide synthase does not prevent the induction of long-term potentiation in vivo, J. Neurosci. 14 (1994) $7415-7425$ 
[3] C.A. Barnes, B.L. McNaughton, D.S. Bredt, C.D. Ferris, S.H. Snyder, Nitric oxide synthase inhibition in vivo has no effect on hippocampal synaptic enhancement of spatial memory, Soc. Neurosci. Abstr. 18 (1992) 1215.

[4] R. Bernabeu, M.L. Destein, C. Fin, I. Izquierdo, J.H. Medina, Role of hippocampal no in the acquisition and consolidation of inhibitory avoidance learning, Neuroreport 6 (1995) 1498-1500.

[5] T.V.P. Bliss, G.L. Collingridge, A synaptic model of memory: Long-term potentiation in the hippocampus, Nature (Lond.) 361 (1993) 31-39.

[6] G.A. Bohme, C. Bon, M. Lemaire, M. Reibaud, O. Piot, J.-M. Stutzman, A. Doble, J.C. Blanchard, Altered synaptic plasticity and memory formation in nitric oxide synthase inhibitor-treated rats, Proc. Natl. Acad. Sci. USA 90 (1993) 9191-9194.

[7] C. Bon, G.A. Bohme, A. Doble, J.-M. Stutzman, J.C. Blanchard, A role for nitric oxide in long-term potentiation, Eur. J. Neurosci. 4 (1992) 420-424.

[8] P.F. Chapman, C.M. Atkins, M.T. Allen, J.E. Haley, J.E. Steinmetz, Inhibition of nitric oxide synthesis impairs two different forms of learning, Neuroreport 3 (1992) 567-570.

[9] H. Daniel, N. Hemart, D. Jaillard, F. Crepel, Long-term depression requires nitric oxide and guanosine $3 ' / 5$ ' cyclic monophosphate production in rat cerebellar Purkinje cells, Eur. J. Neurosci. 5 (1993) 1079-1082.

[10] C. Doyle, C. Holscher, M.J. Rowan, R. Anwyl, The selective neuronal NO synthase inhibitor 7-nitro-indazole blocks both longterm potentiation and depotentiation of field EPSPs in rat hippocampal area CA1 in vivo, J. Neurosci. 16 (1996) 418-424.

[11] E. Dzoljic, R. De Vries, M.R. Dzoljic, New and potent inhibitors of nitric oxide synthase reduce motor activity in mice, Behav. Brain Res. 87 (1997) 209-212.

[12] M.S. Fanselow, Associative vs. topographical accounts of the immediate shock freezing deficit in rats: implications for the response selection rules governing species specific defensive reactions, Learn. Motiv. 17 (1986) 16-39.

[13] M.S. Fanselow, Associations and memories: The role of NMDA receptors and long-term potentiation, Curr. Dir. Psychol. Sci. 2 (1993) 152-156.

[14] J.E. Haley, G.L. Wilcox, P.F. Chapman, The role of nitric oxide in hippocampal long-term potentiation, Neuron 8 (1992) 211-216.

[15] M. Good, R.C. Honey, Dissociable effects of selective lesions to hippocampal subsystems on exploratory behavior, contextual learning, and spatial learning, Behav. Neurosci. 111 (1997) 487-493.

[16] R.D. Hawkins, NO honey, I don't remember, Neuron 16 (1996) 465-467.

[17] C. Holscher, L. McGlinchey, R. Anwyl, M.J. Rowan, 7-Nitro indazole, a selective neuronal nitric oxide synthase inhibitor in vivo, impairs spatial learning in the rat, Learn. Mem. 2 (1996) 267-278.

[18] J.J. Kim, J.P. DeCola, J. Landeira-Fernandez, M.S. Fanselow, $N$ Methyl-D-aspartate receptor antagonist APV blocks acquisition but not expression of fear conditioning, Behav. Neurosci. 105 (1991) $126-133$.

[19] J.J. Kim, R.A. Rison, M.S. Fanselow, Effects of amygdala, hippocampus, and periaqueductal gray lesions on short- and long-term contextual fear, Behav. Neurosci. 107 (1993) 1093-1098.

[20] G.M. Mackenzie, S. Rose, P.A. Bland-Ward, P.K. Moore, P. Jenner, C.D. Marsden, Time course of inhibition of brain nitric oxide synthase by 7-nitro-indazole, Neuroreport 5 (1994) 1993-1996.

[21] S. Maren, M. Baudry, Properties and mechanisms of long-term synaptic plasticity in the mammalian brain: Relationships to learning and memory, Neurobiol. Learn. Mem. 63 (1995) 1-18.

[22] S. Maren, J.P. DeCola, M.S. Fanselow, Water deprivation enhances fear conditioning to contextual, but not discrete, conditional stimuli in rats, Behav. Neurosci. 108 (1994) 645-649.

[23] S. Maren, J.P. DeCola, R.A. Swain, M.S. Fanselow, R.F. Thompson, Parallel augmentation of hippocampal long-term potentiation (LTP), theta rhythm, and contextual fear conditioning in water-deprived rats, Behav. Neurosci. 108 (1994) 44-56.

[24] S. Maren, B. De Oca, M.S. Fanselow, Sex differences in hippocampal long-term potentiation (LTP) and Pavlovian fear conditioning in rats: Positive correlation between LTP and contextual learning, Brain Res. 661 (1994) 25-34.

[25] S. Maren, M.S. Fanselow, Electrolytic lesions of the fimbria/fornix, dorsal hippocampus, or entorhinal cortex produce anterograde deficits in contextual fear conditioning in rats, Neurobiol. Learn. Mem. 67 (1997) 142-149.

[26] S. Maren, G. Aharonov, M.S. Fanselow, Neurotoxic lesions of the dorsal hippocampus and Pavlovian fear conditioning in rats, Behav. Brain Res. 88 (1997) 261-274.

[27] P.K. Moore, P. Wallace, Z. Geffen, S.L. Hart, R.C. Babbedge, Characterization of the novel nitric oxide synthase inhibitor 7-nitro indazole and related indazoles: antinociceptive and cardiovascular effects, Br. J. Pharmacol. 110 (1993) 219-224.

[28] R.G. Phillips, J.E. LeDoux, Lesions of the dorsal hippocampal formation interfere with background by not foreground contextual fear conditioning, Learn. Mem. 1 (1994) 34-44.

[29] T.J. O’Dell, P.L. Huang, T.M. Dawson, J.L. Dinerman, S.H. Snyder, E.R. Kandel, M.C. Fishman, Endothelial NOS and the blockade of LTP by NOS inhibitors in mice lacking neuronal NOS, Science 265 (1994) 542-546.

[30] C. Sandi, C. Venero, C. Guaza, Decreased spontaneous motor-activity and startle response in nitric oxide synthase inhibitor-treated rats, Eur. J. Pharmacol. 277 (1995) 89-97.

[31] E.M. Schuman, D.V. Madison, A requirement for the intercellular messenger nitric oxide in long-term potentiation, Science 254 (1991) $1503-1506$

[32] J.H. Williams, Y.-G. Li, A. Nayak, M.L. Errington, K.P.S.J. Murphy, T.V.P. Bliss, The suppression of long-term potentiation in rat hippocampus by inhibitors of nitric oxide synthase is temperature and age dependent, Neuron 11 (1993) 877-884. 